\title{
Privatsphäre - gefangen im Netz der Koordinaten
}

Am 25. Juni 2009 hat der Landesbeauftragte für Datenschutz und Informationsfreiheit Mecklenburg-Vorpommern gemeinsam mit dem Deutschen Zentrum für Luftund Raumfahrt e. V. Neustrelitz eine Fachtagung unter dem Motto „Privatsphäre gefangen im Netz der Koordinaten“veranstaltet. In der Aula des Neustrelitzer Gymnasiums Carolinum diskutierten ca. 130 Fachleute aus Politik, Wirtschaft, Forschung und Verwaltung über Datenschutzaspekte beim Umgang mit Geodaten in Wirtschaft und Verwaltung.

Der Landesdatenschutzbeauftragte Karsten Neumann eröffnete die Fachtagung mit der Forderung nach einer Allianz aus Recht und Technik, die zu einer Balance zwischen Chancen und Risiken bei der Schaffung einer Geodateninfrastruktur führen müsse.

In seinem Grußwort wies Dr. Stefan Rudolph, Staatssekretär im Ministerium für Wirtschaft, Arbeit und Tourismus des Landes Mecklenburg-Vorpommern, darauf hin, dass ein präventiver Datenschutz wirtschaftlicher ist als ein repressiver Datenschutz. Beim Schutz der Privatsphäre müsse gelten: Agieren ist vernünftiger als Reagieren!

Im ersten Fachvortrag vermittelte Holger Maass vom Deutschen Zentrum für Luftund Raumfahrt e. V. einen Eindruck, welche Möglichkeiten von Informationsprodukten und -diensten bereits heute auf der Grundlage von Satellitensystemen existieren. Am Beispiel von TerraSAR-X stellte er den unmittelbaren Zusammenhang zwischen Sicherheits- und Datenschutzanforderungen dar.

Professorin Lesley Jane Smith, Spezialistin für Fragen des Weltraumrechts, stellte aus der Sicht der Informationsfreiheit die Frage, ob es in der Informationsgesellschaft auch ein grundsätzliches Recht auf Zugang zu Geodaten gäbe. Sie geht davon aus, dass in absehbarer Zeit auf europäischer Ebene Geo-Portale mit öffentlichen Informationen dieses Quasi-Recht verstärkt unterstützen werden. In ihrem Vortrag erörterte Professorin Smith Regulierungsansätze für den Umgang mit Geo-Daten. Diese könn- ten etwa die bereits bestehende Haftung im Sinne der Verantwortlichkeit nach dem Völkerrecht für Erdbeobachtungsinformationen bis hin zur Genehmigungspflicht zur Weitergabe von Geodaten reichen. Neue Lösungen müssen auf der zuständigen europäischen und internationalen Regulierungsebene systematisch angegangen und diskutiert werden.

Dr. Martin Fornefeld, Geschäftsführer der MICUS Management Consulting $\mathrm{GmbH}$, beschrieb den Umsetzungsstand der Richtlinie 2007/2/EG zur Schaffung einer Geodateninfrastruktur in der Europäischen Gemeinschaft (INSPIRE-Richtlinie). Er erläuterte den Zusammenhang der Richtlinie und des daraus folgenden Geodatenzugangsgesetzes mit dem Informationsweiterverwendungsgesetz (IWG), dem Umweltinformationsgesetz (UIG) und dem Informationsfreiheitsgesetzt (IFG).

Dr. Hartmut Streuff aus dem Bundesministerium für Umwelt, Naturschutz und Reaktorsicherheit war innerhalb der Bundesregierung federführend verantwortlich für die Erarbeitung der INSPIRE-Richtlinie. In seinem Vortrag erläuterte er seine Sicht auf die Datenschutzaspekte des neuen Geodatenzugangsgesetzes. Er setzte sich mit der Kritik der Datenschutzbeauftragten von Bund und Ländern am Geodatenzuggangsgesetz auseinander und kam zum Ergebnis, dass entgegen der Auffassung der Datenschützer beim Zugang der Öffentlichkeit zu Geodaten und Geodatendiensten der Schutz personenbezogener Daten schon jetzt angemessen berücksichtigt sei. Dr. Streuff warb für einen möglichst offenen Umgang mit Geodaten und Geodatendiensten, um die Ziele der INSPIRE-Richtlinie (Transparenz, Teilhabe, Verwaltungsvereinfachung, Aktivierung des Wertschöpfungspotenzials) nicht zu gefährden.

Marco Klisch, Leiter des Fachbereiches „Landeskoordinierungsstelle für Geoinformationssysteme " im Landesamt für innere Verwaltung Mecklenburg-Vorpommern, stellte das GeoPortal.MV als zentralen $\mathrm{Zu}-$ gangsknoten zur Geodateninfrastruktur Mecklenburg-Vorpommerns vor. Er zeigte, dass Zugangesberechtigungen mit unter- schiedlichen Sicherungsmechanismen auf Portal-, Netz- und Diensteebene zumindest aus technischer Sicht mit klassischen Rechte- und Rollenmanagementsystem steuerbar sind.

Die Wünsche der Geodatenwirtschaft Deutschlands erläuterte Dr. Jörg Reichling, Leiter der Geschäftsstelle der Kommission für Geoinformationswirtschaft. Er betrachtet Geoinformation als „Digitalen Rohstoff“ für den Wirtschaftsstandort Deutschland. Dr. Reichling geht davon aus, dass nicht nur gute Qualität, hohe Verfügbarkeit, einfacher Zugang und wirtschaftliche Preismodelle sondern auch vernünftiger Datenschutz zu optimierten Geschäftsprozessen und neuen Geschäftsmodellen führen werden.

In seinem Fachbeitrag ging Dr. Thilo Weichert, Landesbeauftragter für den Datenschutz Schleswig-Holstein, auf die immer wieder gestellten Fragen nach dem Personenbezug von Geodaten, den Konsequenzen aus der allgemeinen Zugänglichkeit und dem Interesse an deren Veröffentlichung ein. Bei der Beantwortung dieser Fragen wäre der latente Personenbezug von Geodaten zu berücksichtigen, ohne dass es gerechtfertigt wäre, in jedem Fall die strengen Regeln des allgemeinen Datenschutzrechtes anzuwenden. Dr. Weichert distanzierte sich von der Auffassung des Instituts für Rechtsinformatik (IRI) an der LeibnizUniversität Hannover, das bei der Feststellung des Personenbezugs auf die Zielsetzung der Verarbeitung abstellt. Vielmehr könne die Frage der Anwendung des Datenschutzrechtes nur davon abhängig sein, ob objektiv eine persönlichkeitsrechtliche Gefährdung besteht, nicht ob die verarbeitende Stelle eine solche bejaht.

Karsten Neumann verband sein Schlusswort zur Fachtagung mit dem besonderen Dank an die Gesellschaft für Datenschutz und Datensicherung e. V., die alle Tagungsbeiträge in einem Tagungsband veröffentlichen wird.

Gabriel Schulz, Stellvertreter des Landesbeauftragten für Datenschutz und Informationsfreiheit Mecklenburg-Vorpommern 